\title{
Geodesic systems of tunnels in hyperbolic 3-manifolds
}

\author{
STEPHAN D BURTON \\ JESSICA S PURCELL
}

\begin{abstract}
It is unknown whether an unknotting tunnel is always isotopic to a geodesic in a finite-volume hyperbolic 3-manifold. In this paper, we address the generalization of this question to hyperbolic 3-manifolds admitting tunnel systems. We show that there exist finite-volume hyperbolic 3-manifolds with a single cusp, with a system of $n$ tunnels, $n-1$ of which come arbitrarily close to self-intersecting. This gives evidence that systems of unknotting tunnels may not be isotopic to geodesics in tunnel number $n$ manifolds. In order to show this result, we prove there is a geometrically finite hyperbolic structure on a $(1 ; n)$-compression body with a system of $n$ core tunnels, $n-1$ of which self-intersect.
\end{abstract}

57M50; 57M27, 30F40

\section{Introduction}

One major task in the study of 3-manifolds is to relate topological invariants to geometric ones, for example, to identify arcs with a topological description as geodesics in a hyperbolic manifold. One particular class of arcs that has earned interest is that of unknotting tunnels.

An unknotting tunnel in a 3-manifold $M$ with torus boundary is an embedded arc with endpoints on $\partial M$ whose complement is homeomorphic to a handlebody. A manifold that admits a single unknotting tunnel (and is not a solid torus) is called a tunnel number 1 manifold. A system of unknotting tunnels for a 3-manifold $M$ with torus boundary is a collection of arcs $\tau_{1}, \ldots, \tau_{n}$, each with endpoints on $\partial M$ such that $M \backslash\left(\bigcup_{i=1}^{n} N\left(\tau_{i}\right)\right)$ is a handlebody, where $N(\cdot)$ denotes a regular neighborhood. Manifolds that admit a tunnel system of $n$ arcs but not a tunnel system of $(n-1)$ arcs are called tunnel number $n$. Recall that every 3-manifold with torus boundary is tunnel number $n$ for some $n$, because the tunnel number of the manifold encodes the genus of a minimal genus Heegaard splitting, and every 3-manifold admits a Heegaard splitting. 
Now, unknotting tunnels are defined by topology; they are described by embedded arcs and homeomorphisms. Adams was the first to investigate their geometry in the case that the 3-manifold is hyperbolic [1]. He showed that when $M$ is a tunnel number 1 manifold with two boundary components, then an unknotting tunnel will always be isotopic to a geodesic. He asked if this is true for more general tunnel number 1 manifolds. Soon after, Adams and Reid showed that an unknotting tunnel in a 2-bridge knot complement is always isotopic to a geodesic [2]. Recently, Cooper, Futer and Purcell [8] showed that in an appropriate sense, an unknotting tunnel in a tunnel number 1 manifold is isotopic to a geodesic generically.

In this paper, we investigate the generalization of Adams' question to systems of unknotting tunnels, or tunnel systems, in tunnel number $n$ manifolds and give evidence that the answer to the question is no in this setting. That is, we show that there are tunnel number $n$ manifolds, and a system of $n$ tunnels, such that $(n-1)$ of those tunnels are homotopic to geodesics arbitrarily close to having self-intersections. This is the content of Theorem 1.2, below. Because the geodesics homotopic to these tunnels come within distance $\epsilon$ of self-intersecting, they either must pass through themselves in an attempted isotopy to the tunnel system, or they lie within distance $\epsilon$ of homotopic arcs that pass through themselves under the natural homotopy to the tunnel system. Thus it seems unlikely that all such tunnels will be isotopic to geodesics. Hence this result gives evidence that not all tunnels of all possible tunnel systems for a tunnel number $n$ manifold will be isotopic to geodesics. See below for further discussion.

In order to understand the geometry of tunnel number $n$ manifolds, we study the geometry of compression bodies with genus 1 negative boundary and genus $(n+1)$ positive boundary. We denote these compression bodies as $(1 ; n+1)$-compression bodies. Notice that any tunnel number $n$ manifold with a single torus boundary component is obtained by attaching a genus $(n+1)$ handlebody to a $(1 ; n+1)$-compression body along their common genus $(n+1)$ boundaries. A system of unknotting tunnels in the resulting manifold will consist of a system of arcs in the $(1 ; n+1)$-compression body, which we call core tunnels. In the case of the $(1 ; 2)$-compression body, Lackenby and Purcell investigated the natural extension of Adams' question to geometrically finite hyperbolic structures on such compression bodies [15]. They conjectured that in the $(1 ; 2)$-compression body, core tunnels are always isotopic to geodesics.

Another main result of this paper is that the natural generalization of Lackenby and Purcell's conjecture to $(1 ; 1+n)$-compression bodies is false.

Theorem 1.1 There exist geometrically finite hyperbolic structures on the $(1 ; n+1)-$ compression body for $n \geq 2$, for which $(n-1)$ of the $n$ core tunnels are homotopic to self-intersecting geodesics. Hence these tunnels cannot be isotopic to simple geodesics. 
Theorem 1.1 is obtained by studying Ford domains of geometrically finite structures on such compression bodies. Ford domains have proven useful for the study of geometrically finite structures on manifolds in the past. For example, Jørgensen studied Ford domains of once-punctured torus groups [14] and cyclic groups [13]. Akiyoshi, Sakuma, Wada and Yamashita extended Jørgensen's work [3], and Wada [27] developed an algorithm to determine Ford domains of these manifolds. Lackenby and Purcell also studied Ford domains on $(1 ; 2)$-compression bodies [15], and Ford domains play a role in identifying long tunnels in the work of Cooper, Lackenby and Purcell [9].

Using the Ford domains for geometrically finite hyperbolic structures on $(1 ; n+1)-$ compression bodies as well as geometric techniques to attach handlebodies to such structures as in [9], we show the following.

Theorem 1.2 For any $\epsilon>0$, there exist finite-volume one-cusped hyperbolic manifolds with a system of $n$ tunnels for which $(n-1)$ of the tunnels are homotopic to geodesics that come within distance $\epsilon$ of self-intersecting.

The proof of this theorem does not guarantee that the geodesics will self-intersect. However, the proof involves constructing a sequence of hyperbolic manifolds with geodesics that are close to self-intersecting. In particular, we start with a self-intersecting geodesic and modify the geometry slightly to obtain the new hyperbolic manifold. If the geodesic does not remain self-intersecting under the geometric modification, then it will move in one of two directions, only one of which is in the direction of isotopy of the tunnel. In the other direction, the obvious homotopy to the unknotting tunnel passes through the point of self-intersection, and so is not an isotopy. (In fact, there may still be a non-obvious isotopy even in this case, but the homotopy moving the arc the shortest distance in an $\epsilon$-ball about the nearest points on the geodesic will pass through the geodesic so it will not be an isotopy.) In any case, the geodesic in the homotopy class of the tunnel lies within distance $\epsilon$ of an arc that must pass through a self-intersection in a natural homotopy to the unknotting tunnel. Hence we say these tunnels are "within $\epsilon$ " of not being isotopic to geodesics. This gives evidence that these tunnels are not isotopic to a geodesic, although not a proof of the fact. Moreover, as there are many choices involved in the proof of Theorem 1.2, it is plausible that some choice will produce a hyperbolic manifold with a tunnel system that may have to pass through itself when homotoped to a nearly self-intersecting geodesic. Consequently, it is likely that there are finite-volume tunnel number $n$ manifolds for which $(n-1)$ of the tunnels are not isotopic to a geodesic.

Finally, we note that the proof of this theorem relies upon a specific choice of the spine of a compression body $C$. However, there are countably many choices for any tunnel 
system for tunnel number $n$ manifolds, provided $n \geq 2$. In fact, we will see below that our choice of tunnel systems for Theorem 1.2 is not a natural choice for the geometric structure we start with. In each of our examples, there is a more obvious choice of tunnel systems from a geometric point of view, which leads to a geodesic tunnel system. Therefore, we ask the following.

Question 1.3 For any finite-volume tunnel number $n$ manifold with a single cusp, is there a choice of a system of unknotting tunnels for the manifold such that each tunnel is isotopic to a geodesic?

\section{Acknowledgements}

We thank David Futer, Yair Minsky, Yoav Moriah and Saul Schleimer for helpful conversations. We particularly thank Schleimer for explaining to us the handlebody complex and its application in Section 4.3. Purcell was supported by NSF grant DMS-1007437 and the Alfred P Sloan Foundation.

\section{Background}

In this section, we define notation and review background material on Ford domains of compression bodies. We also prove a few lemmas that will be important later in the paper.

\subsection{The topology of compression bodies}

Here we review topological facts concerning compression bodies. The details are standard and may be found, for example, in Scharlemann's survey article [24]. Complete details on many of the results may be found, among other places, in Burton [6].

Definition 2.1 Let $S$ be a (possibly disconnected) closed, orientable surface with genus at least 1 . A compression body is the result of attaching a finite collection of 1 -handles to $S \times[0,1]$ on the boundary component $S \times\{1\}$, in a piecewise linear manner; we require that our compression bodies be connected.

If $C$ is a compression body, the negative boundary $\partial_{-} C$ is $S \times\{0\}$. The positive boundary $\partial_{+} C$ is $\partial C \backslash \partial_{-} C$.

Note that $\partial_{-} C$ will consist of a disjoint union of surfaces of genus $m_{1}, \ldots, m_{k}$, and $\partial_{+} C$ will be a genus $n$ surface with $n \geq \sum m_{i}$. We will call such a compression body an $\left(m_{1}, \ldots, m_{k} ; n\right)$-compression body. 
In this paper we will only consider examples with $S$ connected, hence we are interested in $(m ; n)$-compression bodies, with $n \geq m$. Usually we will set $m=1$.

Any two $(m ; n)$-compression bodies are homeomorphic [6]. Hence, for fixed $m, n$, we will usually refer to the $(m ; n)$-compression body.

Definition 2.2 A system of disks for a compression body $C$ is a collection of properly embedded essential disks $\left\{D_{1}, \ldots, D_{n}\right\}$ such that the complement of a regular neighborhood of $\bigcup_{i=1}^{n} D_{i}$ in $C$ is homeomorphic to the disjoint union of a collection of balls and the manifold $\partial_{-} C \times[0,1]$. A system of disks is minimal if the complement of a regular neighborhood of $\bigcup_{i=1}^{n} D_{i}$ in $C$ is homeomorphic to $\partial_{-} C \times[0,1]$.

Each $(m ; n)$-compression body admits a minimal system of disks, and such a system of disks will contain exactly $n-m$ disks [6]. In fact, provided $n-m \geq 2$, the $(m ; n)-$ compression body will actually admit countably many systems of disks, each related by some sequence of disk slides as in the following definition.

Definition 2.3 Let $C$ be an $(m ; n)$-compression body with $n-m \geq 2$ and let $\boldsymbol{D}=$ $\left\{D_{1}, \ldots, D_{n-m}\right\}$ be a minimal system of disks for $C$. Let $N$ be a regular neighborhood of $\boldsymbol{D}$. Then $C \backslash N$ is homeomorphic to $\partial_{-} C \times[0,1]$, with the (positive) boundary component $\partial_{-} C \times\{1\}$ containing pairs of disks, denoted $E_{i}$ and $E_{i}^{\prime}$, which are parallel to $D_{i}$.

Let $\alpha$ be an arc in $\partial_{-} C \times\{1\}$ with one endpoint on one disk, say $E_{i}$, and the other endpoint on another disk, say $E_{j}$, and otherwise disjoint from all the disks $E_{k} \cup E_{k}^{\prime}$. Let $N^{\prime}$ be a regular neighborhood in $C$ of $E_{i} \cup \alpha \cup E_{j}$. Then $\bar{N}^{\prime}$ is a closed ball that intersects $\partial_{+} C$ in a thrice-punctured sphere. The set $\partial N^{\prime} \backslash \partial C$ consists of three disks: one parallel to $D_{i}$, one parallel to $D_{j}$ and another disk $D_{i} *_{\alpha} D_{j}$. Let $\boldsymbol{D}^{\prime}=\left\{D_{1}, \ldots, \hat{D}_{i}, \ldots, D_{n}, D_{i} *_{\alpha} D_{j}\right\}$, where as usual $\hat{D}_{i}$ means remove $D_{i}$ from the collection. Then $\boldsymbol{D}^{\prime}$ is also a minimal system of disks. It is said to be a disk slide of $\boldsymbol{D}$. See Figure 1.
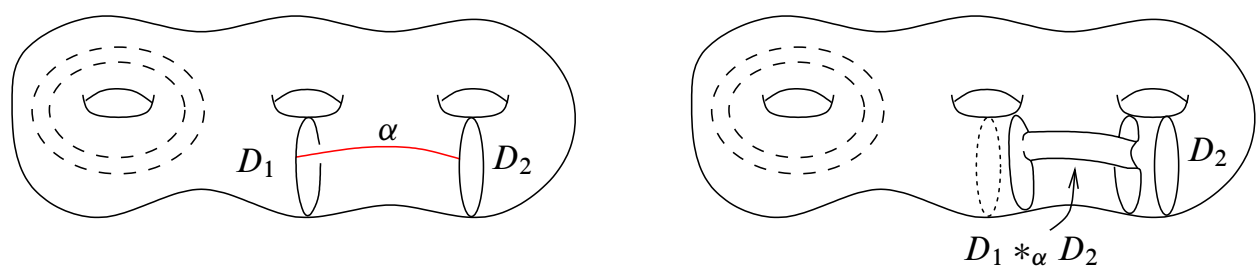

Figure 1: A disk slide in a $(1 ; 3)$-compression body

Associated to a minimal system of disks for $C$ is a system of arcs as follows. 
Definition 2.4 Let $K$ be a graph embedded in a compression body $C$ whose only vertices are valence one, embedded in $\partial_{-} C$. If $C$ deformation retracts to $\partial_{-} C \cup K$, then we say $K$ is a spine for $C$. A spine $K$ is dual to a minimal system of disks $\boldsymbol{D}$ if each edge of $K$ intersects a single disk of $\boldsymbol{D}$ exactly once, and each disk in $\boldsymbol{D}$ intersects an edge of $K$.

Given any minimal system of disks $\boldsymbol{D}$ for a compression body $C$ there is always a spine dual to $\boldsymbol{D}$ and the spine is unique up to isotopy [6].

Definition 2.5 Let $C$ be a compression body and let $K$ be a spine dual to a minimal system of disks $\boldsymbol{D}$ for $C$. The edges of $K$ are arcs running from $\partial_{-} C$ to $\partial_{-} C$. We call such an arc a core tunnel for $C$ and we say the spine $K$ is a core tunnel system, or simply a tunnel system, for $C$.

Just as there are countably many minimal systems of disks $\boldsymbol{D}$ for a compression body $C$, there are also countably many tunnel systems. However, we will work frequently with one particular system, given by the following lemma.

Lemma 2.6 Recall that the $(m ; n)$-compression body $C$ is obtained by attaching $(n-m)$ 1-handles to the $S \times\{1\}$ component of $S \times I$, where $S$ is a genus $m$ surface. For each $i=1, \ldots, n-m$, let $e_{i}$ be an edge at the core of the corresponding 1 -handle, extended vertically through $S \times[0,1]$ to have boundary on $S \times\{0\}$. Then $\bigcup_{i=1}^{n-m} e_{i}$ forms a tunnel system for $C$ and each $e_{i}$ is a core tunnel.

Proof We need to show that $K=\bigcup_{i=1}^{n-m} e_{i}$ is a spine for $C$ that is dual to a minimal system of disks. Note that if we let $D_{i}$ be a cross-sectional disk in a 1-handle, then the collection $\left\{D_{1}, \ldots, D_{n-m}\right\}$ forms a minimal disk system dual to $K$. Moreover, the $i^{\text {th }} 1$-handle deformation retracts to the $e_{i}$ at its core. Extending this to all of $C$, we see that $C$ deformation retracts to $\partial_{-} C \cup K$. Hence each $e_{i}$ is a core tunnel for $C$ and the collection of the $e_{i}$ forms a tunnel system.

Definition 2.7 Inside the $(m ; n)$-compression body $C$, for each core tunnel $e_{i}$ of Lemma 2.6, connect the endpoints of $e_{i}$ by an arc in $S \times\{0\}$. The result is a loop $\gamma_{i}$ in $C$. In fact, if we let $\alpha_{1}, \beta_{1}, \ldots, \alpha_{2 m-2}, \beta_{2 m-2}$ be loops generating $\pi_{1}(S)$, then the loops $\alpha_{j}, \beta_{j}, \gamma_{i}$ for $j=1, \ldots, 2 m-2$ and $i=1, \ldots, n-m$ generate $\pi_{1}(C)$ (after we extend the $\gamma_{i}$ to meet a common basepoint on $S \times\{0\}$ ). We call such a collection of generators standard generators for $\pi_{1}(C)$.

Hereafter, we will primarily work with the $(1 ; n+1)$-compression body. Standard generators will be written as $\alpha, \beta, \gamma_{1}, \ldots, \gamma_{n}$. 


\subsection{Hyperbolic geometry of compression bodies}

We are interested in relating the topology of a compression body $C$ to hyperbolic geometry on $C$. Specifically, we wish to understand the behavior of geodesic arcs homotopic or isotopic to a core tunnel in a hyperbolic structure on the interior of $C$. We obtain a hyperbolic structure by taking a discrete, faithful representation $\rho: \pi_{1}(C) \rightarrow \operatorname{PSL}(2, \mathbb{C})$ and considering the manifold $\mathbb{H}^{3} / \rho\left(\pi_{1}(C)\right)$.

Recall that a discrete subgroup $\Gamma<\operatorname{PSL}(2, \mathbb{C})$ is geometrically finite if $\mathbb{H}^{3} / \Gamma$ admits a finite-sided, convex fundamental domain. In this case, we will also say that the manifold $\mathbb{H}^{3} / \Gamma$ is geometrically finite.

A discrete subgroup $\Gamma<\operatorname{PSL}(2, \mathbb{C})$ is minimally parabolic if it has no rank one parabolic subgroups. In other words, a discrete, faithful representation $\rho: \pi_{1}(M) \rightarrow$ $\operatorname{PSL}(2, \mathbb{C})$ will be minimally parabolic if and only if whenever $\rho(g)$ is parabolic, $g$ is conjugate to an element of the fundamental group of a torus boundary component.

Definition 2.8 A discrete, faithful representation $\rho: \pi_{1}(M) \rightarrow \operatorname{PSL}(2, \mathbb{C})$ is a minimally parabolic geometrically finite uniformization of $M$ if $\rho\left(\pi_{1}(M)\right)$ is minimally parabolic and geometrically finite, and $\mathbb{H}^{3} / \rho\left(\pi_{1}(M)\right)$ is homeomorphic to the interior of $M$.

For any minimally parabolic geometrically finite uniformization $\rho$ of a $(1 ; n+1)-$ compression body, we must describe the Ford domain.

Definition 2.9 Let $A \in \operatorname{PSL}(2, \mathbb{C})$ be loxodromic and let $H$ be any horosphere about infinity in upper half space $\mathbb{H}^{3}$. Then the isometric sphere corresponding to $A$, which we write $I(A)$, is the set of points in $\mathbb{H}^{3}$ equidistant from $H$ and $A^{-1}(H)$.

If $A=\left[\begin{array}{ll}a & b \\ c & d\end{array}\right]$, then it is well known that the isometric sphere $I(A)$ is the Euclidean hemisphere with center $-d / c$ and radius $1 /|c|$ (see, for example [15]).

Definition 2.10 Let $\Gamma$ be a discrete subgroup of $\operatorname{PSL}(2, \mathbb{C})$, with $\Gamma_{\infty}<\Gamma$ the subgroup fixing the point at infinity in $\mathbb{H}^{3}$. For $g \in \Gamma \backslash \Gamma_{\infty}$, let $B_{g}$ denote the open half ball bounded by $I(g)$ and define the equivariant Ford domain $\mathcal{F}$ to be the set

$$
\mathcal{F}=\mathbb{H}^{3} \backslash\left(\bigcup_{g \in \Gamma \backslash \Gamma_{\infty}} B_{g}\right) .
$$

A vertical fundamental domain for a parabolic group $\Gamma_{\infty}$ fixing the point at infinity in $\mathbb{H}^{3}$ is a choice of (connected) fundamental domain for the action of $\Gamma_{\infty}$ that is cut out by finitely many vertical geodesic planes in $\mathbb{H}^{3}$. 
The Ford domain of $\Gamma$ is defined to be the intersection of $\mathcal{F}$ with a vertical fundamental domain for the action of $\Gamma_{\infty}$.

The Ford domain is not canonical, because there is a choice of vertical fundamental domain. However, the region $\mathcal{F}$ is canonical.

Bowditch showed that if $\Gamma<\operatorname{PSL}(2, \mathbb{C})$ is geometrically finite, then every convex fundamental domain for $\mathbb{H}^{3} / \Gamma$ has finitely many faces [4, Proposition 5.7]. In particular, when $\Gamma$ is geometrically finite there will only be finitely many faces in a Ford domain. This means that for all but finitely many elements $g \in \Gamma \backslash \Gamma_{\infty}$ the isometric sphere $I(g)$ is completely covered by some other isometric sphere. We formalize this in a definition.

Definition 2.11 An isometric sphere $I(g)$ is said to be visible if there exists an open set $U \subseteq \mathbb{H}^{3}$ such that $U \cap I(g) \neq \varnothing$, and the hyperbolic distances satisfy

$$
d\left(x, h^{-1}(H)\right) \geq d(x, H)=d\left(x, g^{-1} H\right)
$$

for every $x \in U \cap I(g)$ and $h \in \Gamma \backslash \Gamma_{\infty}$, where $H$ is some horosphere about infinity.

A proof of the following fact may be found in [15].

Lemma 2.12 For $\Gamma$ discrete, the following are equivalent.

(1) The isometric sphere $I(g)$ is visible.

(2) There exists a two dimensional cell of the cell structure on $\mathcal{F}$ contained in $I(g)$.

(3) $I(g)$ is not contained in $\bigcup_{h \in \Gamma \backslash\left(\Gamma_{\infty} \cup \Gamma_{\infty} g\right)} \bar{B}_{h}$.

We will mainly be considering uniformizations of a $(1 ; n+1)$-compression body where the Ford domain is of a particularly simple type, which occurs in the following example.

Example 2.13 Let $C$ be a $(1 ; 3)$-compression body. Then $\pi_{1}(C) \cong(\mathbb{Z} \times \mathbb{Z}) * \mathbb{Z} * \mathbb{Z}$. We will choose generators $\alpha, \beta, \gamma$ and $\delta$ for $\pi_{1}(C)$, where $\alpha$ and $\beta$ generate the $\mathbb{Z} \times \mathbb{Z}$ subgroup. Consider the representation

$$
\begin{array}{ll}
\rho(\alpha)=\left[\begin{array}{cc}
1 & 100 \\
0 & 1
\end{array}\right], & \rho(\beta)=\left[\begin{array}{cc}
1 & 100 i \\
0 & 1
\end{array}\right], \\
\rho(\gamma)=\left[\begin{array}{cc}
0 & 1 \\
-1 & -5 i
\end{array}\right], & \rho(\delta)=\left[\begin{array}{cc}
-5-5 i & -26-25 i \\
1 & 5
\end{array}\right] .
\end{array}
$$

Let $\Gamma_{\infty}=\langle\rho(\alpha), \rho(\beta)\rangle<\operatorname{PSL}(2, \mathbb{C})$. Here we have chosen $\rho(\alpha)$ and $\rho(\beta)$ somewhat arbitrarily so that they give a very large parabolic translation length. Drawing the isometric spheres corresponding to $\rho\left(\gamma^{ \pm 1}\right)$ and $\rho\left(\delta^{ \pm 1}\right)$ gives us the picture in Figure 2. 

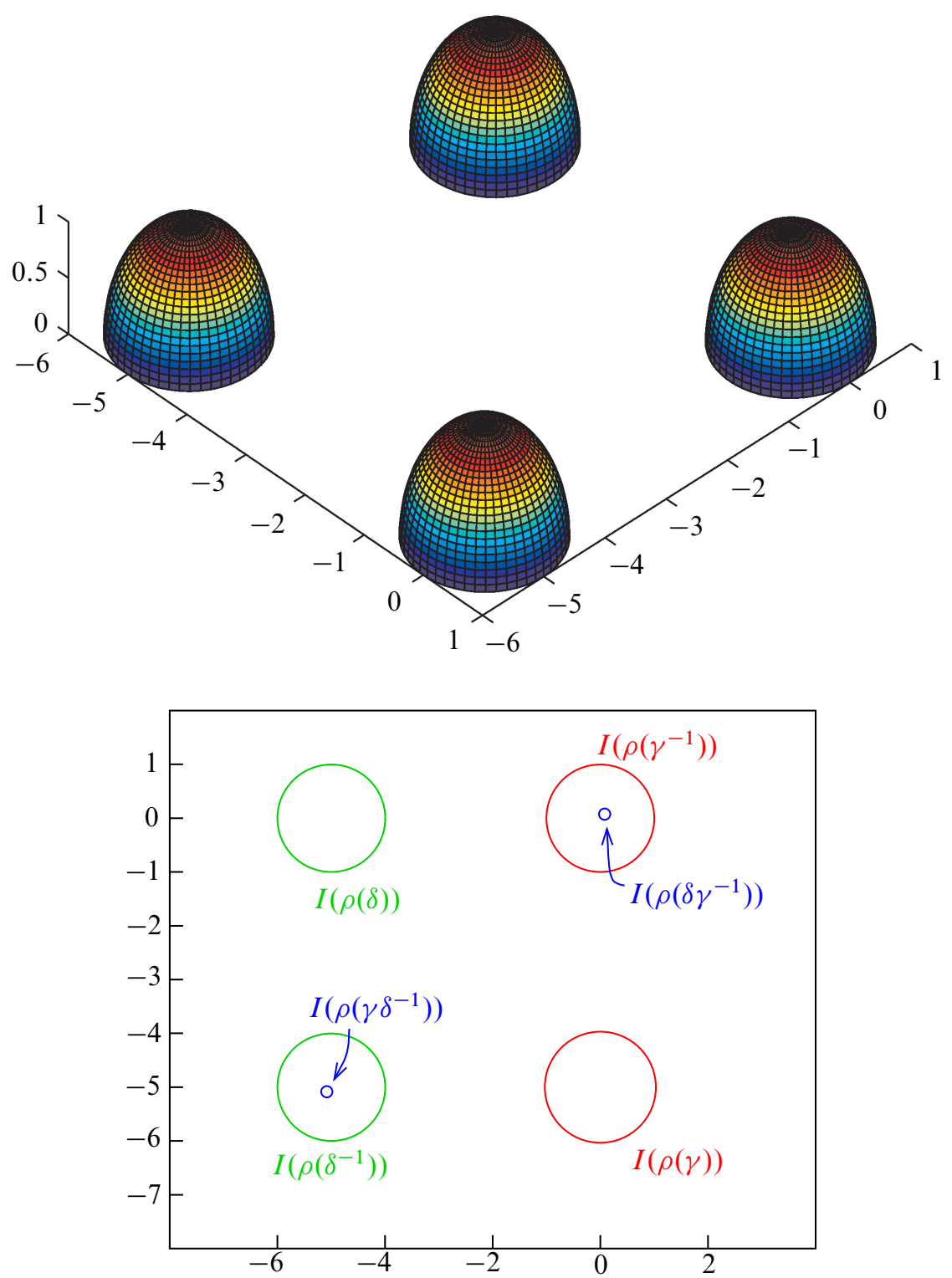

Figure 2: Isometric spheres from Example 2.13 are shown in 3-dimensions at the top and their 2-dimensional intersections with $\mathbb{C}$ at the bottom

We will see that other isometric spheres, besides the translates under $\Gamma_{\infty}$ of $I\left(\rho\left(\gamma^{ \pm 1}\right)\right)$ and $I\left(\rho\left(\delta^{ \pm 1}\right)\right)$, will be invisible, hidden underneath these isometric spheres. For example, the isometric spheres $I\left(\rho\left(\gamma \delta^{-1}\right)\right)$ and $I\left(\rho\left(\delta \gamma^{-1}\right)\right)$ shown in Figure 2 are 
invisible. Hence $\rho$ will give a minimally parabolic geometrically finite uniformization of $C$ whose Ford domain is as in Figure 2.

We now generalize Example 2.13. To do so, set up the following notation.

Let $C_{n}$ denote the $(1 ; n+1)$-compression body. Hence $\pi_{1}\left(C_{n}\right) \cong(\mathbb{Z} \times \mathbb{Z}) * F_{n}$, where $F_{n}$ denotes the free group on $n$ letters $\mathbb{Z} * \cdots * \mathbb{Z}$. Let $\alpha, \beta, \gamma_{1}, \ldots, \gamma_{n}$ be generators of $\pi_{1}\left(C_{n}\right)$, with $\alpha$ and $\beta$ generating the $(\mathbb{Z} \times \mathbb{Z})$ subgroup and $\gamma_{1}, \ldots, \gamma_{n}$ standard as in Definition 2.7, coming from a tunnel system of $C_{n}$ as in Lemma 2.6. Finally, let $\rho: \pi_{1}\left(C_{n}\right) \rightarrow \operatorname{PSL}(2, \mathbb{C})$ be a discrete representation, taking $\alpha$ and $\beta$ to parabolics fixing infinity, generating the subgroup $\Gamma_{\infty}=\langle\rho(\alpha), \rho(\beta)\rangle<\operatorname{PSL}(2, \mathbb{C})$.

Definition 2.14 With notation as above, suppose the isometric spheres corresponding to $\rho\left(\gamma_{1}^{ \pm 1}\right), \ldots, \rho\left(\gamma_{n}^{ \pm 1}\right)$ and their translates under $\Gamma_{\infty}$ are all pairwise disjoint, with none properly contained in a half-ball bounded by one of the others. Then we say that $\rho$ gives a simple Ford domain for $C_{n}$.

Note that Example 2.13 is an example of a simple Ford domain. The use of the words "Ford domain" in Definition 2.14 is justified by the following lemma.

Lemma 2.15 Suppose $\rho: \pi_{1}\left(C_{n}\right) \rightarrow \operatorname{PSL}(2, \mathbb{C})$ gives a simple Ford domain for $C_{n}$. Then $\rho$ gives a minimally parabolic, geometrically finite uniformization of $C_{n}$. Moreover, after possibly replacing the $\gamma_{i}$ by multiples of $\gamma_{i}$ with elements in the $(\mathbb{Z} \times \mathbb{Z})$ subgroup of $\pi_{1}\left(C_{n}\right)$, the isometric spheres corresponding to $\rho\left(\gamma_{1}^{ \pm 1}\right), \ldots, \rho\left(\gamma_{n}^{ \pm 1}\right)$, along with a choice of vertical fundamental domain for $\Gamma_{\infty}$, cut out a Ford domain.

Proof Choose a vertical fundamental domain for $\Gamma_{\infty}$. Recall that the center of the isometric sphere $I\left(\rho\left(\gamma_{i}\right)\right)$ lies at the point $\rho\left(\gamma_{i}^{-1}\right)(\infty)$. We may multiply each $\gamma_{i}$, $i=1, \ldots, n$, on the right by some $w_{i} \in(\mathbb{Z} \times \mathbb{Z})$ so that the center $\rho\left(w_{i}^{-1} \gamma_{i}^{-1}\right)(\infty)$ of the isometric sphere $I\left(\rho\left(\gamma_{i} w_{i}\right)\right)$ lies inside the chosen vertical fundamental domain. Note that $w_{i}$ is a word in $\alpha$ and $\beta$, and so $\alpha, \beta, \gamma_{1} w_{1}, \ldots, \gamma_{n} w_{n}$ still generate $\pi_{1}\left(C_{n}\right)$, and the $\gamma_{i} w_{i}$ still give isometric spheres whose translates under $\Gamma_{\infty}$ are pairwise disjoint as in Definition 2.14. Thus without loss of generality, we may assume that the centers of the $I\left(\rho\left(\gamma_{i}\right)\right)$ are all contained in our chosen vertical fundamental domain.

Next, consider the isometric spheres corresponding to $\rho\left(\gamma_{i}^{-1}\right)$ for $i=1, \ldots, n$. We may multiply each $\gamma_{i}, i=1, \ldots, n$, on the left by some $x_{i} \in(\mathbb{Z} \times \mathbb{Z})$ so that the center $\rho\left(x_{i}^{-1} \gamma_{i}\right)(\infty)$ of the isometric sphere $I\left(\rho\left(\gamma_{i}^{-1} x_{i}\right)\right)$ lies inside the chosen vertical fundamental domain. Note also that the center $\rho\left(\gamma_{i}^{-1} x_{i}\right)(\infty)$ of $I\left(\rho\left(x_{i}^{-1} \gamma_{i}\right)\right)$ is the same as the center $\rho\left(\gamma_{i}^{-1}\right)(\infty)$ of $I\left(\rho\left(\gamma_{i}\right)\right)$, because $\rho\left(x_{i}\right)$ fixes $\infty$, so when we 
replace each $\gamma_{i}$ by $x_{i}^{-1} \gamma_{i}$ we obtain generators of $\pi_{1}\left(C_{n}\right)$ such that the corresponding isometric spheres $I\left(\rho\left(\gamma_{i}^{ \pm 1}\right)\right)$ all have centers within our chosen vertical fundamental domain. Moreover, note that these isometric spheres still satisfy the definition of a simple Ford domain.

Now, let $P$ be the intersection of the chosen vertical fundamental domain with the exterior of the isometric spheres corresponding to $\rho\left(\gamma_{i}^{ \pm 1}\right)$. Because none of these isometric spheres is contained inside another and because they do not intersect, $P$ is homeomorphic to a 3-ball, marked with simply connected faces, and these faces correspond to the faces of the vertical fundamental domain and to each isometric sphere $I\left(\rho\left(\gamma^{ \pm 1}\right)\right)$.

Identify vertical sides of $P$ by elements of $\Gamma_{\infty}$ and glue $I\left(\rho\left(\gamma_{i}\right)\right)$ to $I\left(\rho\left(\gamma_{i}^{-1}\right)\right)$ via $\rho\left(\gamma_{i}^{-1}\right)$ for each $i=1, \ldots, n$. This glues faces of $P$ by isometry and since the intersections of faces (edges of $P$ ) are only on the vertical fundamental domain, the Poincaré polyhedron theorem (cf Lackenby and Purcell [15, Theorem 2.21] and Epstein and Petronio [11]) implies that the result of applying these gluings to $P$ is a smooth manifold $M$, with $\pi_{1}(M) \cong \pi_{1}\left(C_{n}\right)$ generated by face pairings. Moreover, by [15, Theorem 2.22], $P$ must be a Ford domain for $M \cong \mathbb{H}^{3} / \Gamma$ and by [15, Lemma 2.18], it is minimally parabolic.

Hence, to show that this gives a minimally parabolic geometrically finite uniformization of $C_{n}$, it remains only to show that $M$ is homeomorphic to $C_{n}$. We show this by considering gluing faces of $P$ one at a time.

First, glue faces corresponding to the vertical fundamental domain. Since $\Gamma_{\infty}$ is a rank2 parabolic group, the result is homeomorphic to $T^{2} \times \mathbb{R}$, where $T^{2}$ is the torus. Now, notice that when we glue the face $I\left(\rho\left(\gamma_{i}\right)\right)$ to $I\left(\rho\left(\gamma_{i}^{-1}\right)\right)$, the result is topologically equivalent to attaching a 1 -handle. Hence, when performing the gluing one by one for each $i=1, \ldots, n$, we obtain a manifold homeomorphic to $C_{n}$.

\subsection{Tunnel systems and hyperbolic geometry}

We are interested in studying a tunnel system for a manifold, and we need to identify a tunnel system in a geometrically finite minimally parabolic uniformization.

For $\gamma \in \operatorname{PSL}(2, \mathbb{C})$ that does not fix the point at infinity in $\mathbb{H}^{3}$, there is a geodesic $e_{\gamma}$ running from $\gamma^{-1}(\infty)$ to $\infty$ in $\mathbb{H}^{3}$. This geodesic $e_{\gamma}$ will meet the center of the Euclidean hemisphere $I(\gamma)$. We say that $e_{\gamma}$ is the geometric dual of the isometric sphere $I(\gamma)$. We also refer to $e_{\gamma}$ as the geodesic dual to the isometric sphere $I(\gamma)$. 
Lemma 2.16 Let $C$ denote the $(1 ; n+1)$-compression body, where $\pi_{1}(C) \cong$ $(\mathbb{Z} \times \mathbb{Z}) * F_{n}$ has generators $\alpha, \beta, \gamma_{1}, \ldots, \gamma_{n}$, with $\alpha$ and $\beta$ generating the $(\mathbb{Z} \times \mathbb{Z})$ subgroup and $\gamma_{1}, \ldots, \gamma_{n}$ standard, coming from a tunnel system as in Definition 2.7. Let $\rho: \pi_{1}(C) \rightarrow \operatorname{PSL}(2, \mathbb{C})$ be a minimally parabolic, geometrically finite uniformization of $C$, normalized such that $\rho(\alpha)$ and $\rho(\beta)$ fix the point of infinity of $\mathbb{H}^{3}$. Finally, let $\tilde{d}_{i}$ be the geodesic dual to the isometric sphere $I\left(\rho\left(\gamma_{i}^{-1}\right)\right)$. Then under the quotient action of $\Gamma$, the images of the dual edges $\tilde{d}_{i}$ are homotopic to a spine of $C$. Hence these geometric edges are homotopic to a tunnel system.

Proof We will show that the images of the geodesics $\tilde{d}_{i}$ are homotopic to the core tunnels $e_{i}$ of Lemma 2.6, and this will be enough to prove the lemma.

In the topological manifold $C$, take a regular neighborhood $N$ of $\partial_{-} C$ so that the closure $\bar{N}$ is homeomorphic to $\partial_{-} C \times[0,1]$. Choose $p=\left(p^{\prime}, 1\right) \in \partial_{-} C \times\{1\}$ and let $q=\left(p^{\prime}, 0\right) \in \partial_{-} C \times\{0\}$. Let $f:[0,1] \rightarrow C$ be the straight line from $p$ to $q$.

In $\mathbb{H}^{3}$, choose a vertical fundamental domain $D$ for $\Gamma_{\infty}=\langle\rho(\alpha), \rho(\beta)\rangle$. As in the proof of Lemma 2.15, we may replace the $\gamma_{i}$ by products $w_{i} \cdot \gamma_{i} \cdot v_{i}$, where $w_{i}, v_{i}$ are in $\Gamma_{\infty}$, and thereby assume that $D$ contains $\rho\left(\gamma_{i}^{ \pm 1}\right)(\infty)$ for all $i=1,2, \ldots, n$ (or rather, these points are contained in the closure of $D$ in $\left.\mathbb{H}^{3} \cup \mathbb{C} \cup\{\infty\}\right)$. Note that the replacement doesn't affect the argument, since under these translations dual geodesics $\tilde{d}_{i}$ still map to the same geodesic in $\mathbb{H}^{3} / \rho\left(\pi_{1}(C)\right)$.

The lift $\tilde{p}$ of $p$ into $D$ is a point on a horoball $H$ about $\infty$. For each loxodromic $\rho\left(\gamma_{i}\right)$, define $\tilde{p}_{i}=\rho\left(\gamma_{i}\right)(\tilde{p})$. The point $\tilde{p}_{i}$ lies on a horosphere centered at $\rho\left(\gamma_{i}\right)(\infty)$. For each $i=1,2, \ldots, n$, let $\tilde{g}_{i}$ be a geodesic arc in $D$ from $\tilde{p}$ to $\tilde{p}_{i}$. Under the action of $\Gamma$, the arc $\tilde{g}_{i}$ becomes a loop in the homotopy class of $\gamma_{i}$ in $C$.

Let $\tilde{f}_{i}$ be a geodesic arc in $D$ from $\tilde{p}_{i}$ to $\gamma_{i}(\infty)$ and let $\widetilde{f}_{i}^{\prime}$ be a geodesic arc from $\infty$ to $\tilde{p}$. Under the action of $\Gamma$, the arcs $\tilde{f}_{i}$ and $\tilde{f}_{i}^{\prime}$ are mapped to $\operatorname{arcs}$ in $C$ from $p$ to points on $\partial_{-} C$, these arcs are homotopic to $f$ rel $p$, and the homotopy may be taken to keep an endpoint of each of the arcs on $\partial_{-} C$.

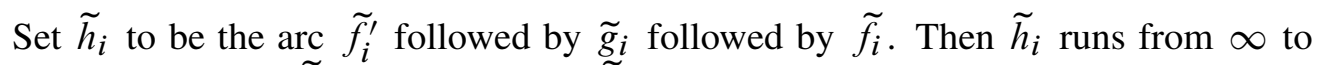
$\gamma_{i}(\infty)$. Therefore $\tilde{h}_{i}$ is homotopic to $\widetilde{d}_{i}$.

On the other hand, under the action of $\Gamma, \tilde{h}_{i}$ is mapped to a loop with endpoints on $\partial_{-} C$ in the homotopy class of $\gamma_{i}$. Allowing the endpoints of this loop to move on $\partial_{-} C$, we may homotope to the arc $e_{i}$, which is a core tunnel from Lemma 2.6, corresponding to the standard generator $\gamma_{i}$ coming from Definition 2.7.

Lemma 2.16 shows only that the geodesic duals to isometric spheres corresponding to a set of generators gives a set homotopic to a tunnel system. We are interested in 
examples of when these geodesics are isotopic to a tunnel system. One example of when this will occur comes from the following lemma.

Lemma 2.17 With notation as in Lemma 2.16, if the Ford domain is simple and the isometric sphere corresponding to each $\rho\left(\gamma_{i}\right)$ is visible, then in the quotient manifold $C \cong \mathbb{H}^{3} / \rho\left(\pi_{1}(C)\right)$ the images of the $\widetilde{d}_{i}$ are isotopic to a spine of $C$. Hence these edges form a geodesic tunnel system.

Proof Let $\mathcal{F}$ be the equivariant Ford domain. Let $H \subset \mathcal{F}$ be an embedded horoball about infinity. As in [15, Lemma 3.11], we construct an equivariant deformation retract of $\mathcal{F} \backslash H$ onto the union of the geodesic $\operatorname{arcs} \Gamma_{\infty}\left(\tilde{d}_{i} \cap(\mathcal{F} \backslash H)\right)$ and $\partial H$. We do so in two steps.

First, by Lemma 2.15, we may assume that the isometric spheres corresponding to $\rho\left(\gamma_{i}^{ \pm 1}\right), i=1, \ldots, n$, along with a vertical fundamental domain for $\Gamma_{\infty}$ cut out a Ford domain. The boundaries of the isometric spheres give embedded circles on $\mathbb{C}$ which bound disjoint disks $D_{1}, D_{1}^{\prime}, D_{2}, D_{2}^{\prime}, \ldots, D_{n}, D_{n}^{\prime}$ on $\mathbb{C}$, with $D_{i}$ corresponding to $\rho\left(\gamma_{i}\right)$ and $D_{i}^{\prime}$ corresponding to $\rho\left(\gamma_{i}^{-1}\right)$. Now, choose a value of $\epsilon>0$ such that for the disks $E_{i}$ and $E_{i}^{\prime}$, which are the $\epsilon$ neighborhoods of $D_{i}$ and $D_{i}^{\prime}$, respectively, the collection $E_{1}, E_{1}^{\prime}, E_{2}, E_{2}^{\prime}, \ldots, E_{n}, E_{n}^{\prime}$ on $\mathbb{C}$ still consists of disjoint disks. Take the vertical projection of these $E_{i}, E_{i}^{\prime}$ onto the boundary of the horoball $H$; we will continue to denote these disks on $\partial H$ by $E_{1}, E_{1}^{\prime}, \ldots, E_{n}, E_{n}^{\prime}$. For each $i=1,2, \ldots, n$, consider the frustum $C_{i}$ of the solid (Euclidean) cone in $\mathbb{H}^{3}$ that intersects $\partial H$ in the disk $E_{i}$ and intersects $\mathbb{C}$ in the disk $D_{i}$. Similarly, we have the frustum $C_{i}^{\prime}$ meeting $\partial H$ in $E_{i}^{\prime}$ and meeting $\mathbb{C}$ in $D_{i}^{\prime}$. By choice of $\epsilon$, the sets $C_{1} \cap(\mathcal{F} \backslash H)$, $C_{1}^{\prime} \cap(\mathcal{F} \backslash H), \ldots, C_{n} \cap(\mathcal{F} \backslash H), C_{n}^{\prime} \cap(\mathcal{F} \backslash H)$, as well as their translates under $\Gamma_{\infty}$, are disjoint in $\mathcal{F} \backslash H$. Let

$$
C=\Gamma_{\infty}\left(\bigcup_{i=1}^{n}\left(C_{i} \cup C_{i}^{\prime}\right) \cap(\mathcal{F} \backslash H)\right) \subset(\mathcal{F} \backslash H) .
$$

The first step of the homotopy is to map $\mathcal{F} \backslash(C \cup H)$ onto $\partial(C \cup H)$ via the vertical line homotopy. That is, each point $x$ in $\mathcal{F} \backslash(C \cup H)$ lies on a vertical line through $\infty$, and this line will meet $\partial(C \cup H)$ exactly once. Let $L_{t}(x)$ be the point on this vertical line, so that $L_{0}(x)$ is the identity and $L_{1}(x)$ lies on $\partial(C \cup H)$. Note the map $L_{t}$ is continuous, equivariant under the action of $\rho\left(\pi_{1}(C)\right)$, and descends to a continuous map in the quotient $\mathbb{H}^{3} / \rho\left(\pi_{1}(C)\right)$.

The second step is to deformation retract $C \cap(\mathcal{F} \backslash H)$ onto the set

$$
\left(\partial H \cup\left(\bigcup_{i=1}^{n} \Gamma_{\infty}\left(\tilde{d}_{i} \cup \rho\left(\gamma_{i}\right)\left(\tilde{d}_{i}\right)\right)\right)\right) \cap(\mathcal{F} \backslash H) .
$$


Since $C_{i}$ and $C_{i}^{\prime}$ form regular neighborhoods of $\tilde{d}_{i}$ and $\rho\left(\gamma_{i}\right)\left(\tilde{d}_{i}\right)$, respectively, there is a deformation retract sending each $C_{i} \cap(\mathcal{F} \backslash H)$ and $C_{i}^{\prime} \cap(\mathcal{F} \backslash H), i=1, \ldots, n$, onto the geodesic at its core. Note by choice of $\epsilon$, we may perform these deformation retracts simultaneously and equivariantly, since none of these cones intersect in $\mathcal{F} \backslash H$. It is clear that we can modify this deformation retract to a deformation retract onto $\tilde{d}_{i} \cup\left(\partial H \cap C_{i}\right)$ or $\rho\left(\gamma_{i}\right) \tilde{d}_{i} \cup\left(\partial H \cap C_{i}^{\prime}\right)$ for $i=1, \ldots, n$. We let $f_{t}$ be the deformation retract of the second step. Then the deformation retract $L_{t}$ followed by $f_{t}$ is the desired equivariant deformation retract.

\section{Tunnel systems in compression bodies}

In this section we show that the geodesic duals in the Ford domain may be made to intersect while retaining a geometrically finite structure.

Lemma 3.1 Let $\gamma$ and $\delta$ be loxodromic generators of a $(1 ; n)$-compression body $C$. Suppose that under some geometrically finite uniformization $\rho: \pi_{1}(C) \rightarrow \operatorname{PSL}(2, \mathbb{C})$ of $C$, the faces of the Ford domain corresponding to $\rho\left(\delta^{ \pm 1}\right)$ and $\rho\left(\left(\delta \gamma^{-1}\right)^{ \pm 1}\right)$ are visible, and that the isometric sphere $I(\rho(\gamma))$ is contained in the Euclidean half-ball bounded by the isometric sphere $I(\rho(\delta))$. Then the geometric dual $\tilde{g}$ to $I(\rho(\gamma))$ in $\mathbb{H}^{3}$ is mapped to a geodesic $g$ under the quotient $\mathbb{H}^{3} \rightarrow \mathbb{H}^{3} / \rho\left(\pi_{1}(C)\right)$ with the property that $g$ lifts to geodesics in $\mathbb{H}^{3}$ containing the arcs:

(1) $\alpha_{1}$, running from $\infty$ to a point on $I(\rho(\delta)$ ) (a subarc of the geodesic dual to $I(\rho(\gamma)))$,

(2) $\alpha_{2}$, running from a point on $I\left(\rho\left(\delta^{-1}\right)\right)$ to a point on $I\left(\rho\left(\gamma \delta^{-1}\right)\right.$ ) (a subarc of the geodesic from the center of $I\left(\rho\left(\delta^{-1}\right)\right)$ to the center of $\left.I\left(\rho\left(\gamma \delta^{-1}\right)\right)\right)$ and

(3) $\alpha_{3}$, running from $\infty$ to a point on $I\left(\rho\left(\delta \gamma^{-1}\right)\right.$ ) (a subarc of the geodesic dual to $\left.I\left(\rho\left(\gamma^{-1}\right)\right)\right)$.

Lemma 3.1 is illustrated in Figure 3.

Proof Since the uniformization $\rho: \pi_{1}(C) \rightarrow \operatorname{PSL}(2, \mathbb{C})$ is applied to each group element in the proof we will suppress it for ease of notation, writing $\gamma$, for example, rather than $\rho(\gamma)$.

Choose a horosphere $H$ about $\infty$. Let $S$ be the set of points in $\mathbb{H}^{3}$ equidistant from $\delta^{-1}(H)$ and $\gamma^{-1}(H)$. Let $p_{1}$ be the intersection of $I(\delta)$ and $\tilde{g}$, and let $p_{2}$ be the intersection of $S$ and $\tilde{g}$. Note that $p_{2}$ is contained inside the Euclidean half-ball bounded by $I(\delta)$, since $I(\gamma)$ is contained inside that half-ball. 


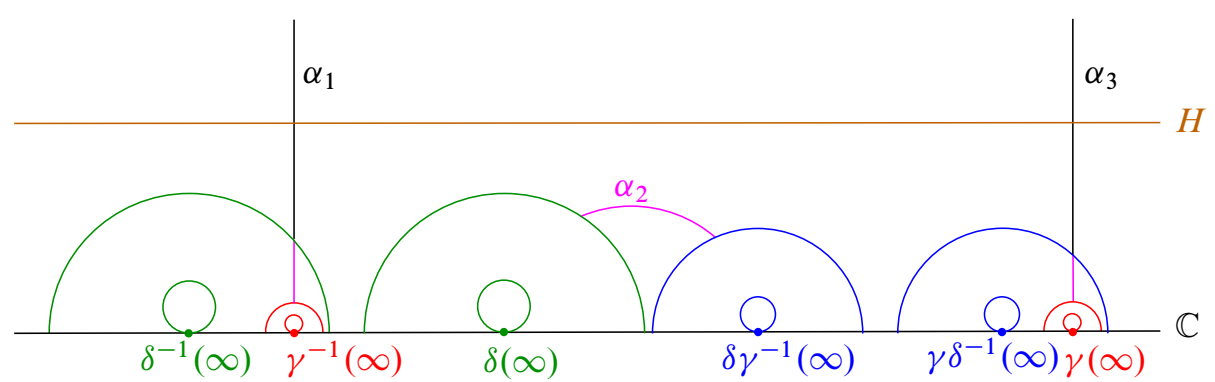

Figure 3: Lifts of $g$ consists of the arcs $\alpha_{1}, \alpha_{2}$, and $\alpha_{3}$

Now apply $\delta$ to this picture. Under $\delta$, the horosphere $\delta^{-1}(H)$ is mapped to $H$ and $H$ is mapped to $\delta(H)$, and so the isometric sphere is $I(\delta)$ mapped to $I\left(\delta^{-1}\right)$ isometrically. Likewise, $S$ gets mapped isometrically to $I\left(\gamma \delta^{-1}\right)$. The geodesic dual $\tilde{g}$ is mapped to the geodesic running from $\delta(\infty)$ to $\delta \gamma^{-1}(\infty)$. These are exactly the centers of the isometric spheres $I\left(\delta^{-1}\right)$ and $I\left(\gamma \delta^{-1}\right)$, respectively. Now $\delta(\widetilde{g})$ is a geodesic that passes through $\delta\left(p_{1}\right) \in I\left(\delta^{-1}\right)$ and $\delta\left(p_{2}\right) \in I\left(\gamma \delta^{-1}\right)$.

In a similar manner as above, apply $\gamma$. The isometric sphere $I(\gamma)$ is mapped to $I\left(\gamma^{-1}\right)$ and $S$ is mapped to $I\left(\delta \gamma^{-1}\right)$. The geodesic dual $\tilde{g}$ gets mapped to the geodesic dual to $I\left(\gamma^{-1}\right)$. Therefore $\tilde{g}$ gets mapped to an arc containing the vertical line from a point on $I\left(\gamma^{-1}\right)$ to $\infty$.

Now $\gamma(\widetilde{g}), \delta(\widetilde{g})$ and $\tilde{g}$ are all mapped to the same geodesic $g$ in the quotient $\mathbb{H}^{3} / \rho\left(\pi_{1}(C)\right)$. The arcs $\alpha_{1}, \alpha_{2}$ and $\alpha_{3}$ are just the portions of these geodesics that lie above $I\left(\delta^{ \pm 1}\right)$ and $I\left(\left(\delta \gamma^{-1}\right)^{ \pm 1}\right)$.

Proposition 3.2 There exists a geometrically finite, minimally parabolic uniformization $\rho$ of a $(1 ; 3)$-compression body $C$, and a generator $\xi$ of the free part of $\pi_{1}(C)$ such that the image of the geometric dual to $I(\rho(\xi))$ under the action of $\rho\left(\pi_{1}(C)\right)$ has a self-intersection.

Proof We prove this by giving a specific example. Recall that $\pi_{1}(C) \cong(\mathbb{Z} \times \mathbb{Z}) * \mathbb{Z} * \mathbb{Z}$. We will let $\alpha, \beta, \gamma_{1}$ and $\gamma_{2}$ generate $\pi_{1}(C)$, such that $\alpha$ and $\beta$ generate the $\mathbb{Z} \times \mathbb{Z}$ subgroup.

We will consider a family of representations $\rho_{t}: \pi_{1}(C) \rightarrow \operatorname{PSL}(2, \mathbb{C})$ for which $\rho_{t}(\alpha)$, $\rho_{t}(\beta)$ and $\rho_{t}\left(\gamma_{1}\right)$ are constant and $\rho_{t}\left(\gamma_{2}\right)$ varies. For this example,

$$
\rho_{t}(\alpha)=\left[\begin{array}{cc}
1 & 20 \\
0 & 1
\end{array}\right], \quad \rho_{t}(\beta)=\left[\begin{array}{cc}
20 i & 1 \\
0 & 1
\end{array}\right] .
$$


These values of $\rho_{t}(\alpha)$ and $\rho_{t}(\beta)$ are chosen so that the translation distances are large, basically so that we can ignore the effect of these two elements on the changing Ford domain.

We let $B$ be the translation matrix $B=\left[\begin{array}{ll}1 & 10 \\ 0 & 1\end{array}\right]$.

We obtain $\rho_{t}\left(\gamma_{1}\right)$ by conjugation by $B$ and we set $\rho_{t}\left(\gamma_{2}\right)$ to vary with $t$ :

$$
\rho\left(\gamma_{1}\right)=B\left[\begin{array}{cc}
0 & 1 \\
-1 & 5-2 i
\end{array}\right] B^{-1}, \quad \rho_{t}\left(\gamma_{2}\right)=\left[\begin{array}{cc}
0 & 1 \\
-1 & 5+(t-2) i
\end{array}\right]
$$

Note that the isometric spheres corresponding to $\rho_{t}\left(\gamma_{1}\right), \rho_{t}\left(\gamma_{1}^{-1}\right), \rho_{t}\left(\gamma_{2}\right)$ and $\rho_{t}\left(\gamma_{2}^{-1}\right)$ all have radius 1 and centers at $10,15-2 i, 0$ and $5+(t-2) i$, respectively. Hence for all $t \in[0,4]$, none of these isometric spheres intersect. Similarly, since the translation distances of $\rho_{t}(\alpha)$ and $\rho_{t}(\beta)$ are large, no translates of these isometric spheres under $\Gamma_{\infty}=\left\langle\rho_{t}(\alpha), \rho_{t}(\beta)\right\rangle$ will intersect. So a vertical fundamental domain as well as isometric spheres corresponding to $\rho_{t}\left(\gamma_{1}^{ \pm 1}\right)$ and $\rho_{t}\left(\gamma_{2}^{ \pm 1}\right)$ cut out a simple Ford domain, and by Lemma 2.15 , this is a minimally parabolic geometrically finite uniformization for $C$.

Now set $\delta_{1}=\gamma_{1}$ and $\delta_{2}=\gamma_{2}^{-1} \gamma_{1}$. Then $\alpha, \beta, \delta_{1}, \delta_{2}$ generate $\pi_{1}(C)$. Moreover, the isometric sphere corresponding to $\rho_{t}\left(\delta_{2}\right)$ will be contained in the Euclidean half-ball bounded by the isometric sphere corresponding to $\rho_{t}\left(\delta_{1}\right)$. See Figure 4 .
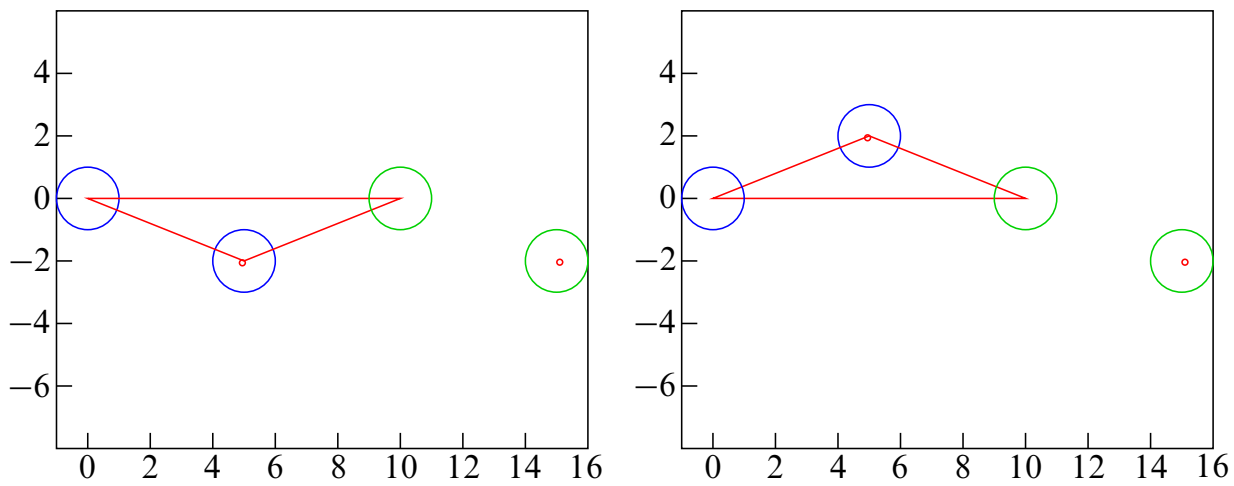

Figure 4: When $t=0$, the Ford domain is as pictured on the left. When $t=4$, the Ford domain is as pictured on the right.

Hence we have exactly the setup of Lemma 3.1, with $\delta_{1}$ playing the role of $\delta$ and $\delta_{2}$ playing the role of $\gamma$. Thus under the action of $\rho_{t}\left(\pi_{1}(C)\right)$, a portion of the geodesic dual to the isometric sphere of $\rho\left(\delta_{2}\right)$ is mapped to a geodesic running from a point 
$p_{1}(t)$ on the isometric sphere of $\rho_{t}\left(\delta_{1}^{-1}\right)=\rho\left(\gamma_{1}^{-1}\right)$ to a point $p_{2}(t)$ on the isometric sphere of $\rho_{t}\left(\delta_{2} \delta_{1}^{-1}\right)=\rho_{t}\left(\gamma_{2}^{-1}\right)$.

Define $p_{3}(t)$ to be the intersection of the geodesic dual to the isometric sphere of $\rho_{t}\left(\delta_{2}^{-1}\right)$ with the isometric sphere of $\rho_{t}\left(\gamma_{2}\right)$. For each $t$ define a Euclidean triangle $T_{t}$ whose vertices are the projections of $p_{1}(t), p_{2}(t)$ and $p_{3}(t)$ onto $\mathbb{C}$.

Let the function $A:[0,4] \rightarrow \mathbb{R}$ give the signed area of $T_{t}$. Carefully,

$$
A(t)=\frac{1}{2}\left(\left(p_{1}(t)-p_{3}(t)\right) \times\left(p_{2}(t)-p_{3}(t)\right)\right),
$$

where $p_{i}(t)-p_{j}(t)$ is understood to be the 3 -dimensional Euclidean vector whose first two coordinates come from the 2 -dimensional subtraction and whose $3^{\text {rd }}$ coordinate is zero, and $\times$ denotes the usual Euclidean cross product on $\mathbb{R}^{3}$. Because the points $p_{1}(t), p_{2}(t)$ and $p_{3}(t)$ vary continuously with $t, A(t)$ is a continuous function.

As can be seen in Figure 4, when $t=0$ we have $A(0)>0$ since $p_{3}(0)$ must be below the line segment from $p_{1}(0)$ to $p_{2}(0)$. When $t=4$ we obtain $A(4)<0$, since $p_{3}(4)$ must be above the line segment. The intermediate value theorem guarantees that there is some $t_{0} \in[0,4]$ for which $A\left(t_{0}\right)=0$, ie $p_{1}\left(t_{0}\right), p_{2}\left(t_{0}\right)$ and $p_{3}\left(t_{0}\right)$ are collinear. Hence when $t=t_{0}$, two of the geodesic arcs guaranteed by Lemma 3.1 will intersect. Thus the image of the geometric dual to the isometric sphere of $\rho_{t_{0}}\left(\delta_{2}\right)$, under the action of $\rho_{t_{0}}\left(\pi_{1}(C)\right)$, will have a self-intersection.

We may generalize Proposition 3.2 to $(1 ; n+1)$-compression bodies. The following result is Theorem 1.1 from the introduction, restated.

Theorem 3.3 There exists a geometrically finite, minimally parabolic uniformization $\rho$ of a $(1 ; n+1)$-compression body $C_{n}$ and a choice of free generators $\delta_{1}, \ldots, \delta_{n}$ of $\pi_{1}\left(C_{n}\right)$ such that the geodesics $\tau_{1}, \ldots, \tau_{n-1}$ obtained from the geometric duals to isometric spheres corresponding to $\rho\left(\delta_{1}\right), \ldots, \rho\left(\delta_{n-1}\right)$, respectively, each self-intersect.

Proof As above, let $\alpha, \beta, \gamma_{1}, \ldots, \gamma_{n}$ generate $\pi_{1}\left(C_{n}\right)$ with $\alpha$ and $\beta$ generating the $\mathbb{Z} \times \mathbb{Z}$ subgroup. Set $A=\left[\begin{array}{ll}1 & 10 \\ 0 & 1\end{array}\right]$.

Let $t=\left(t_{1}, \ldots, t_{n}\right) \in[0,4] \times \cdots \times[0,4] \times\{2\}$ and consider the $n$-parameter family of representations

$$
\begin{gathered}
\rho_{t}\left(\gamma_{k}\right)=A^{k-1}\left[\begin{array}{cc}
0 & 1 \\
-1 & 5+\left(t_{k}-2\right) i
\end{array}\right] A^{-(k-1)} \text { for } 1 \leq k \leq n, \\
\rho_{t}(\alpha)=\left[\begin{array}{cc}
1 & 11 n \\
0 & 1
\end{array}\right], \quad \rho_{t}(\beta)=\left[\begin{array}{cc}
1 & 10 i \\
0 & 1
\end{array}\right] .
\end{gathered}
$$


Note the isometric sphere corresponding to $\rho_{t}\left(\gamma_{k}^{-1}\right)$ has radius 1 and center $10(k-1)$. The isometric sphere corresponding to $\rho_{t}\left(\gamma_{k}\right)$ also has radius 1 and center

$$
5+10(k-1)+\left(t_{k}-2\right) i .
$$

Hence for $t \in[0,4] \times \cdots \times[0,4] \times\{2\}$, all these isometric spheres are disjoint. Moreover, $\rho_{t}(\alpha)$ and $\rho_{t}(\beta)$ are chosen to be large enough so that the parabolic translates of these isometric spheres do not intersect. Thus a vertical fundamental domain as well as the isometric spheres corresponding to $\rho_{g}\left(\gamma_{k}^{ \pm 1}\right)$ cut out a simple Ford domain, and this is a minimally parabolic geometrically finite uniformization for $C_{n}$.

Set $\delta_{k}=\gamma_{k}^{-1} \gamma_{n}$ for $1 \leq k<n$ and $\delta_{n}=\gamma_{n}$. The elements $\delta_{1}, \ldots, \delta_{n}, \alpha, \beta$ still generate $\pi_{1}(C)$, but for $1 \leq k<n$ the isometric sphere corresponding to $\delta_{k}^{-1}$ is not visible: it is contained in the Euclidean half-ball bounded by the isometric sphere corresponding to $\gamma_{k}$. Similarly, for $1 \leq k<n$, the isometric sphere corresponding to $\delta_{k}$ is contained in the Euclidean half-ball bounded by the isometric sphere corresponding to $\gamma_{n}$. Thus Lemma 3.1 implies that the geodesic running from the center of $I\left(\rho_{t}\left(\gamma_{k}^{-1}\right)\right)$ to the center of $I\left(\rho_{t}\left(\gamma_{n}^{-1}\right)\right)$ maps to the image of the geodesic dual to $I\left(\rho_{t}\left(\delta_{k}\right)\right)$.

Now apply a similar argument to that in the previous proof. The intermediate value theorem implies that for each $k, 1 \leq k<n$, there must be a $t_{k} \in[0,4]$ such that the geodesic dual to $I\left(\rho_{t}\left(\delta_{k}\right)\right)$ has self-intersecting image. Because for $i \neq k$, varying $t_{k}$ has no effect on isometric spheres corresponding to $\delta_{k}^{ \pm 1}$, we may perform the above procedure for each $k$ one at a time to obtain the desired uniformization.

\section{Finite-volume tunnel number $\boldsymbol{n}$ manifolds}

In this section, we use the results of the previous section to give evidence that there exist tunnel number $n$ manifolds with finite volume and tunnel systems that come arbitrarily close to self-intersecting.

The rough idea of the proof is to take the compression body of Theorem 3.3 and attach a handlebody to it in such a way that the geometry of the compression body after attaching is "close" to the geometry before attaching. This is accomplished in a manner similar to that of Cooper, Lackenby and Purcell in [9].

\subsection{Maximally cusped structures}

We recall definitions and results on maximally cusped geometrically finite structures, because we will use these structures to build manifolds with nearly self-intersecting tunnels. 
Definition 4.1 A maximally cusped structure for $C$ is a geometrically finite uniformization $\rho: \pi_{1}(C) \rightarrow \operatorname{PSL}(2, \mathbb{C})$ of $C$ such that every component of the boundary of the convex core of $\mathbb{H}^{3} / \rho\left(\pi_{1}(C)\right)$ is a 3 -punctured sphere.

In a maximally cusped structure for $C$, a full pants decomposition of $\partial_{+} C$ is pinched to parabolic elements. A theorem of Canary, Culler, Hersonsky and Shalen [7], extending work of McMullen [21], shows that the conjugacy classes of maximally cusped structures for $C$ are dense on the boundary of all geometrically finite structures on $C$. To make this statement more precise, we review the following definitions.

Definition 4.2 The representation variety $V(C)$ of a compression body $C$ is the space of conjugacy classes of representations $\rho: \pi_{1}(C) \rightarrow \operatorname{PSL}(2, \mathbb{C})$, where $\rho$ sends elements of $\pi_{1}\left(\partial_{-} C\right)$ to parabolics. (This definition is similar to one given by Marden in [19] and is more restrictive than one found in Culler and Shalen [10].) Convergence in $V(C)$ is defined by algebraic convergence. We denote the subset of conjugacy classes of minimally parabolic geometrically finite uniformizations of $C$ by $G F_{0}(C) \subseteq V(C)$. We will give $G F_{0}(C)$ the algebraic topology. Marden [18] showed that $G F_{0}(C)$ is open in $V(C)$.

By [7], conjugacy classes of maximally cusped structures are dense in the boundary of $G F_{0}(C)$ in $V(C)$.

Now, we need to recognize indiscrete representations $\rho: \pi_{1}(C) \rightarrow \operatorname{PSL}(2, \mathbb{C})$. The following lemma, which is essentially the Shimizu-Leutbecher lemma (Maskit [20, Proposition II.C.5]), allows us to do so. A proof using the notation of this paper can be found in [9].

Lemma 4.3 Let $\Gamma$ be a discrete torsion free subgroup of PSL(2, $\mathbb{C})$ such that $M=$ $\mathbb{H}^{3} / \Gamma$ has a rank two cusp. Suppose the point at $\infty$ projects to the cusp and $\Gamma_{\infty} \leq \Gamma$ is the subgroup of parabolics fixing $\infty$. Then for every $\gamma \in \Gamma \backslash \Gamma_{\infty}$ the isometric sphere $I(\gamma)$ has radius at most $T$, where $T$ is the minimal Euclidean translation length of all elements of $\Gamma_{\infty}$.

Using the above lemma, we can show the following.

Lemma 4.4 For any $\epsilon>0$, there exists a maximally cusped structure on the $(1, n+1)-$ compression body $C$, a system of core tunnels $\tau_{1}, \ldots, \tau_{n}$ for $C$ and balls of radius $\epsilon$, $B_{1}(\epsilon), \ldots, B_{n-1}(\epsilon)$ such that for $i=1, \ldots, n-1$, the tunnel $\tau_{i}$ intersects the ball $B_{i}(\epsilon)$ in two distinct arcs. Hence $n-1$ of the $n$ tunnels come within distance at most $\epsilon$ of self-intersecting. 
Proof Let $\rho_{0}$ be the geometrically finite representation of the $(1, n+1)$-compression body constructed in Theorem 3.3, with generators $\alpha, \beta, \delta_{1}, \ldots, \delta_{n}$ for $\pi_{1}(C)$ and where the geodesic duals to the isometric spheres corresponding to $\rho_{0}\left(\delta_{1}\right), \ldots, \rho_{0}\left(\delta_{n-1}\right)$ glue up to have self-intersections. We need to recall a bit more detail about where the intersection occurs. Recall from the proof of Theorem 3.3 that in the universal cover $\mathbb{H}^{3}$, for $i=1, \ldots, n-1$, the geodesic dual to the isometric sphere $I\left(\rho_{0}\left(\delta_{i}\right)\right)$ intersects the geodesic running from the center of the isometric sphere $I\left(\rho_{0}\left(\delta_{i} \delta_{n}^{-1}\right)\right)$ to the center of the isometric sphere $I\left(\rho_{0}\left(\delta_{n}^{-1}\right)\right)$, and that these two geodesics have the same image in $\mathbb{H}^{3} / \rho_{0}\left(\pi_{1}(C)\right)$.

Now, the translation lengths of $\rho_{0}(\alpha)$ and $\rho_{0}(\beta)$ are bounded by some number $L$. We can consider $\rho_{0}$ as an element of $V(C)$. Let $\mathcal{R}$ be the set of all representations $\rho$ of $\pi_{1}(C)$ where $\rho(\alpha), \rho(\beta)$ are parabolics fixing infinity with translation length bounded by $L$ and $\rho\left(\delta_{i}\right)=\rho_{0}\left(\delta_{i}\right)$. By suitably normalizing $\rho(\alpha), \rho(\beta)$ to avoid conjugation, we can view $\mathcal{R}$ as a subset of $V(C)$. Note that $\rho_{0} \in \mathcal{R}$.

Also note that for any geometrically finite structure $\rho$ in $\mathcal{R}$ and for all $i=1, \ldots, n-1$, the geodesic arc dual to the isometric sphere $I\left(\rho\left(\delta_{i}\right)\right)$ will intersect the geodesic running from the center of $I\left(\rho\left(\delta_{i} \delta_{n}^{-1}\right)\right)$ to the center of $I\left(\rho\left(\delta_{n}^{-1}\right)\right)$, and again these geodesics have the same image in $\mathbb{H}^{3} / \rho\left(\pi_{1}(C)\right)$, giving self-intersecting tunnels in each of these structures.

Now, there exists a path in $\mathcal{R}$ from $\rho_{0}$ to some indiscrete representation. Such a path is obtained by decreasing the minimal translation length of $\rho(\alpha)$ or $\rho(\beta)$ until it becomes smaller than the radius of an isometric sphere. This gives an indiscrete structure by Lemma 4.3. Hence this path intersects $\partial G F_{0}(C)$ at some point, say $\rho_{\infty}$.

Maximally cusped structures are dense in $\partial G F_{0}(C)$ [7]. Hence there exists a sequence of geometrically finite representations $\rho_{k}$ of $\pi_{1}(C)$ such that the conformal boundaries of the manifolds $C_{k}=\mathbb{H}^{3} / \rho_{k}\left(\pi_{1}(C)\right)$ are maximally cusped genus $(n+1)$ surfaces, each $C_{k}$ is homeomorphic to the interior of $C$ and the algebraic limit of the $\rho_{k}$ is $\rho_{\infty}$.

Now, for each $\delta_{i}, i=1, \ldots, n, \rho_{k}\left(\delta_{i}\right)$ converges to $\rho_{\infty}\left(\delta_{i}\right)$, hence the center of the corresponding isometric sphere $I\left(\rho_{k}\left(\delta_{i}\right)\right)$ converges to the center of the isometric sphere $I\left(\rho_{\infty}\left(\delta_{i}\right)\right)$. Similarly, the centers of the isometric spheres $I\left(\rho_{k}\left(\delta_{i} \delta_{n}^{-1}\right)\right)$ and $I\left(\rho_{k}\left(\delta_{n}^{-1}\right)\right)$ converge to the centers of the isometric spheres $I\left(\rho_{\infty}\left(\delta_{i} \delta_{n}^{-1}\right)\right)$ and $I\left(\rho_{\infty}\left(\delta_{n}^{-1}\right)\right)$, respectively.

Hence for any $\epsilon>0$, there exists $K>0$ such that if $k>K$, the geodesic $\tau_{k}$ dual to $I\left(\rho_{k}\left(\delta_{i}\right)\right)$, and its image $\rho_{k}\left(\delta_{i}^{-1} \delta_{n}\right)\left(\tau_{k}\right)$, with endpoints at the centers of isometric spheres $I\left(\rho_{k}\left(\delta_{i} \delta_{n}^{-1}\right)\right)$ and $I\left(\rho_{k}\left(\delta_{n}^{-1}\right)\right)$, are within distance $\epsilon / 2$ of each other. Let $p_{i}$ be a point of distance at most $\epsilon / 4$ from both geodesics. Note the two geodesics 
intersect the ball $B_{\epsilon}\left(p_{i}\right)$ of radius $\epsilon$ in $\mathbb{H}^{3}$. Let $B_{i}(\epsilon)$ denote the image of the ball $B_{\epsilon}\left(p_{i}\right)$ in the quotient manifold $\mathbb{H}^{3} / \rho_{k}\left(\pi_{1}(C)\right)$. The image of the geodesic $\tau_{i}$ runs through $B_{i}(\epsilon)$ in two distinct arcs as desired.

Lemma 4.5 For any $\epsilon>0$ and any integer $n \geq 2$, there exists a finite volume hyperbolic 3-manifold $M$ with the following properties.

(1) $M$ is obtained from a manifold $\widehat{M}$ with genus $(n+1)$ Heegaard surface $S$ by drilling out a collection of curves on $S$ corresponding to a full pants decomposition of $S$.

(2) There exists a tunnel system $\tau_{1}, \ldots, \tau_{n}$ for $\widehat{M}$ and balls $B_{1}(\epsilon), \ldots, B_{n-1}(\epsilon) \subset$ $M$ such that for each $i=1, \ldots, n-1$, the geodesic arc in the homotopy class of $\tau_{i}$ in $M$ intersects $B_{i}(\epsilon)$ in at least two nontrivial arcs. Hence the arc comes within $\epsilon$ of self-intersecting in $M$.

Proof Let $C_{D}$ be a maximally cusped compression body of Lemma 4.4. The collection of curves on the conformal boundary of $C_{D}$ forms a pants decomposition $P$ of the genus $(n+1)$ surface.

Let $H$ be a genus $(n+1)$ handlebody. We wish to take a maximally cusped hyperbolic structure on $H$ for which the rank-1 cusps on $\partial H$ consist exactly of the curves of $P$. In fact, there are infinitely many such structures, which follows as a consequence of Thurston's uniformization theorem (see Morgan [22]). Let $H_{D}$ denote one such structure.

Now, consider the convex cores of $C_{D}$ and of $H_{D}$. The boundaries of the convex cores consist of 3-punctured spheres, which have a unique hyperbolic structure. Hence we may glue $\partial_{+} C_{D}$ to $\partial H_{D}$ via isometry on each 3 -punctured sphere, and we obtain a finite volume hyperbolic 3-manifold $M$ with $(3 n+1)$ rank-2 cusps. One of these cusps comes from the rank-2 cusp of $C_{D}$. The other $3 n$ come from gluing together the $3 n$ rank-1 cusps on the boundaries of the convex cores of $C_{D}$ and $H_{D}$.

Note that if we do a trivial Dehn filling of the $3 n$ cusps of $M$ that came from rank1 cusps on $\partial_{+} C_{D}$ and $\partial H_{D}$, then we obtain a manifold $\widehat{M}$ with a genus $(n+1)$ Heegaard splitting along a surface we denote $S$. This gives item (1).

The core tunnels $\tau_{1}, \ldots, \tau_{n}$ of $C_{D}$ become a system of tunnels for $\widehat{M}$ under the gluing. Because the gluing is by isometry and the balls $B_{i}(\epsilon)$ of Lemma 4.4 lie within the convex core of $C_{D}$ for $i=1, \ldots, n-1$, the tunnels $\tau_{1}, \ldots, \tau_{n-1}$ satisfy item (2). 


\subsection{Filling the manifold}

In order to obtain a manifold with a tunnel system consisting of arcs arbitrarily close to self-intersecting, we will take the cusped manifold of Lemma 4.5 and perform Dehn filling on the $3 n$ cusps corresponding to the pants decompositions of the surface $S$.

Lemma 4.6 Let $M$ be the manifold of Lemma 4.5 and let $S$ be the surface in item (1) of that lemma. So $M$ is homeomorphic to the interior of a compact manifold $\bar{M}$ with torus boundary components $T_{1}, \ldots, T_{3 n+1}$ and $S \cap M$ intersects $\bar{M}$ in a surface $\bar{S}$ with boundary on $T_{1}, \ldots, T_{3 n}$. For each torus boundary component $T_{j}, j=1, \ldots, 3 n$, we take a basis for $H_{1}\left(T_{j}\right)$ consisting of the curves $\lambda_{j}, \mu_{j}$, where $\lambda_{j}$ is a component of $\partial \bar{S} \cap T_{j}$ and $\mu_{j}$ is any curve with intersection number 1 with $\lambda_{j}$. Then Dehn filling $\bar{M}$ along any slope $\mu_{j}+k \lambda_{j}$ will yield a manifold with Heegaard surface $S$.

Proof Any such slope has intersection number one with the surface $S$. It is well known that Dehn filling on such a slope acts as a Dehn twist on the surface $S$, and the $S$ is a Heegaard surface for every such Dehn filling (see Lickorish [16] and Rolfsen [23]).

We are now ready to prove Theorem 1.2 from the introduction, which we restate.

Theorem 4.7 For any $\epsilon>0$ and any integer $n \geq 2$, there exists a finite volume hyperbolic 3-manifold $M$ with a single cusp torus such that $M$ has the following property. It admits a system of tunnels $\left\{\tau_{1}, \ldots, \tau_{n}\right\}$ and a collection of balls $B_{1}(\epsilon), \ldots, B_{n-1}(\epsilon)$ of radius $\epsilon$ such that for $i=1, \ldots, n-1$, the geodesic arc homotopic to $\tau_{i}$ intersects $B_{i}(\epsilon)$ in two distinct arcs.

In other words, the tunnels $\tau_{1}, \ldots, \tau_{n-1}$ have geodesic representatives that come arbitrarily close to self-intersecting. Although the proof does not guarantee that these tunnels do self-intersect, it gives evidence that there exist tunnels that are not isotopic to geodesics.

Proof Let $M$ be the manifold of Lemma 4.5, say with $\epsilon$ replaced by $\epsilon / 4$ in that lemma. By Lemma 4.6, Dehn filling the cusps of $M$ corresponding to the pants curves of $\widehat{M}$ along slopes $\mu_{j}+k_{j} \lambda_{j}$ will yield a manifold with a genus $(n+1)$ Heegaard splitting.

By work of Thurston [26], as $k_{j}$ approaches infinity the Dehn filling approaches the manifold $M$ in the Gromov-Hausdorff topology. Even more precisely, work of Brock and Bromberg [5] implies that for any $\epsilon_{1}>0$, if $k_{j}$ is large enough, there is a 
$\left(1+\epsilon_{1}\right)$-bilipschitz diffeomorphism $\phi$ from the complement of a Margulis tube about the unfilled cusp to the complement of a Margulis tube about the core of the filled solid torus. We may take these Margulis tubes to avoid the tunnels of our tunnel system. Moreover, $\phi$ is level preserving in the unfilled cusp. Hence for large enough $k_{j}$, we obtain the desired result.

\subsection{Tunnel number $n$}

It would be nice to add to the conclusions of Theorem 4.7 that $M$ is tunnel number $n$, and not some lower tunnel number. Since for a manifold $M$ with one torus boundary component a system of $n$ tunnels corresponds to a genus $n+1$ Heegaard splitting, we can prove that our manifold is tunnel number $n$ by showing there are no lower genus Heegaard splittings of $M$. If the Hempel distance of the Heegaard splitting is high, then work of Scharlemann and Tomova [25] will imply that there are no lower genus Heegaard splittings. Hence in this section we will review Hempel distance and other results on the curve complex that will allow us to conclude our manifold is tunnel number $n$. It should be noted that the final step in this procedure relies on announced work of Maher and Schleimer [17], which as of yet has not appeared. Hence we include the result in a separate section.

Definition 4.8 Let $S$ be a closed, oriented, connected surface. The curve complex $\mathcal{C}(S)$ is the simplicial complex whose vertices are isotopy classes of essential curves in $S$ and a collection of $k+1$ vertices form a $k$-simplex whenever the corresponding curves can be realized by disjoint curves on $S$. For $\alpha, \beta$ vertices in $C(S)$, we define the distance $d_{S}(\alpha, \beta)$ to be the minimal number of edges in any path in the 1 -skeleton of $\mathcal{C}(S)$ between $\alpha$ and $\beta$.

The disk set of a compression body with outer boundary homeomorphic to $S$ is defined to consist of vertices in $\mathcal{C}(S)$ which are the boundaries of essential disks in the compression body. Similarly, the disk set of a handlebody with boundary $S$ consists of vertices of $\mathcal{C}(S)$ which are boundaries of essential disks in the handlebody. Note the disk set of a compression body with outer boundary $S$ is contained in the disk set of a handlebody with boundary $S$.

Definition 4.9 A Heegaard splitting of a 3-manifold along a surface $S$ has two disk sets, one on either side of $S$. The Hempel distance of the Heegaard splitting is defined to be the minimal distance in $\mathcal{C}(S)$ between those disk sets. See Hempel [12].

More generally, we define the inner distance between sets $A$ and $B$ in $\mathcal{C}(S)$ to be

$$
d(A, B)=\inf \left\{d_{S}(a, b) \mid a \in A, b \in B\right\} .
$$


Thus the Hempel distance is the inner distance between the disk sets on either side of a Heegaard surface.

Scharlemann and Tomova showed that if the Hempel distance of a genus $g$ Heegaard splitting is strictly greater than $2 g$, then the manifold will have a unique Heegaard splitting of genus $g$ and no Heegaard splittings of smaller genus [25].

Definition 4.10 For any closed, oriented, connected surface $S$, let the handlebody graph $\mathcal{H}(S)$ be the graph that has a vertex for each handlebody with boundary $S$. Since any handlebody has an associated disk set, alternately we may think of $\mathcal{H}(S)$ as having vertices corresponding to disk sets in $\mathcal{C}(S)$. There is an edge in $\mathcal{H}(S)$ between two handlebodies whose disk sets intersect in the curve complex $\mathcal{C}(S)$. The distance $d_{H}(x, y)$ between any two handlebodies in $\mathcal{H}(S)$ is defined to be the minimal number of edges in a path between them in $\mathcal{H}(S)$.

There is a relation $D: \mathcal{H}(S) \rightarrow \mathcal{C}(S)$ defined as follows. For a handlebody $V \in \mathcal{H}(S)$, $D(V)$ consists of the disk set of $V$. The following lemma was pointed out to us by S Schleimer.

Lemma 4.11 For any handlebodies $V$ and $W$ in $\mathcal{H}(S)$,

$$
d_{H}(V, W) \leq d(D(V), D(W))+1 .
$$

(The right-hand side is inner distance in the curve complex.)

Proof Given handlebodies $V$ and $W$, let $\alpha \in D(V)$ and $\beta \in D(W)$ with $d_{S}(\alpha, \beta)=$ $k$. Take a minimal length path in $\mathcal{C}(S)$ between $\alpha$ and $\beta$ and denote the vertices of the path by $\alpha=\alpha_{0}, \alpha_{1}, \ldots, \alpha_{k}=\beta$. Note that for any $i=1,2, \ldots, k$, there exists a handlebody that we denote $V_{i}$ such that $\alpha_{i-1}$ and $\alpha_{i}$ both bound essential disks in $V_{i}$. Then we obtain a sequence of handlebodies $V_{1}, V_{2}, \ldots, V_{k}$ with $d_{H}\left(V_{i}, V_{i+1}\right)=1$. Since $d_{H}\left(V, V_{1}\right)=1=d_{H}\left(V_{k}, W\right)$, the sequence of handlebodies $V, V_{1}, \ldots, V_{k}, W$ gives a path from $V$ to $W$ of length $k+1$ in $\mathcal{H}(S)$. Hence $d_{H}(V, W) \leq d_{S}(\alpha, \beta)+1$. Since $\alpha$ and $\beta$ were arbitrary,

$$
d_{H}(V, W)-1 \leq \inf \left\{d_{S}(\alpha, \beta) \mid \alpha \in D(V), \beta \in D(W)\right\}=d(D(V), D(W))
$$

as desired.

Maher and Schleimer have proved that $\mathcal{H}(S)$ has infinite diameter [17]. We will use this to prove the following strengthened version of Theorem 4.7. 
Theorem 4.12 For any $\epsilon>0$ and any integer $n \geq 2$, there exists a finite volume hyperbolic tunnel number $n$ manifold $M$ with a single cusp torus such that $M$ has the following property. It admits a system of tunnels $\left\{\tau_{1}, \ldots, \tau_{n}\right\}$ and a collection of balls $B_{1}(\epsilon), \ldots, B_{n-1}(\epsilon)$ of radius $\epsilon$ such that for $i=1, \ldots, n-1$, the geodesic arc homotopic to $\tau_{i}$ intersects $B_{i}(\epsilon)$ in two distinct arcs.

The difference between this theorem and Theorem 4.7 is that here we may conclude that our manifold is tunnel number $n$, while there we just have a system of $n$ tunnels.

Proof As in the proof of Theorem 4.7, we will start with the maximally cusped compression body of Lemma 4.4, attach to it a maximally cusped handlebody and Dehn fill in such a way that the resulting manifold is geometrically close to the original. However, we will choose the maximally cusped structure on our handlebody more carefully to ensure that after Dehn filling, the Hempel distance of the resulting Heegaard splitting remains high.

We first set up notation. Let $C_{0}$ be the maximally cusped structure on the $(1 ; n+1)-$ compression body from Lemma 4.4. Let $S$ denote the positive boundary of this compression body. Let $D\left(C_{0}\right)$ denote the disk set of $C_{0}$ in $\mathcal{C}(S)$. The pinched curves on $S$ corresponding to the rank-1 cusps of $C_{0}$ form a maximal simplex $P$ in $\mathcal{C}(S)$.

Notice that there is a relation $h$ from disk sets of a compression body with outer boundary $S$ to the handlebody complex $\mathcal{H}(S)$ as follows. For a disk set $D$ of a compression body, $h(D)$ consists of all $V \in \mathcal{H}(S)$ for which $D$ is a subset of $D(V)$. So in particular, $h\left(D\left(C_{0}\right)\right)$ is a subset of $\mathcal{H}(S)$.

Recall that a multitwist along $P$ is a collection of Dehn twists, one along each curve of $P$. Let $X=\left\{h\left(T\left(D\left(C_{0}\right)\right)\right) \mid T\right.$ is a multitwist along $\left.P\right\}$. That is, $X$ is the subset of $\mathcal{H}(S)$ consisting of all handlebodies whose disk sets contain $T\left(D\left(C_{0}\right)\right)$ for some multitwist $T$.

Now for the next step of the proof, we show that $X$ has bounded diameter in $\mathcal{H}(S)$.

First, recall that a Dehn twist along any curve in $P$ is an isometry of $\mathcal{C}(S)$, fixing $P$ pointwise. Thus, if we let $K$ denote the inner distance between $P$ and $D\left(C_{0}\right)$ in $\mathcal{C}(S)$ and if we let $T$ be any multitwist along $P$, then $T\left(D\left(C_{0}\right)\right)$ has inner distance $K$ from $P$.

Now, for any $V, W \in X$, by definition of $X$ there exist multitwists $T_{1}$ and $T_{2}$ such that $T_{1}\left(D\left(C_{0}\right)\right) \subset D(V)$ and $T_{2}\left(D\left(C_{0}\right)\right) \subset D(W)$. Then the inner distance $d(D(V), D(W))$ satisfies

$$
\begin{aligned}
d(D(V), D(W)) & \leq d(D(V), P)+d(P, D(W)) \\
& \leq d\left(T_{1}\left(D\left(C_{0}\right)\right), P\right)+d\left(P, T_{2}\left(D\left(C_{0}\right)\right)\right)=2 K .
\end{aligned}
$$


By Lemma 4.11, $d_{H}(V, W) \leq 2 K+1$. Hence $X$ has bounded diameter in $\mathcal{H}(S)$.

We are finally ready to choose the maximally cusped structure on our handlebody. Since the diameter of $\mathcal{H}(S)$ is infinite [17], we may choose a handlebody $Y$ in $\mathcal{H}(S)$ such that $\inf \left\{d_{H}(Y, V) \mid V \in X\right\}=N$, where $N$ is some number, at least $2 n+3$. Let $H_{0}$ be a maximally cusped structure on $Y$ with the curves $P$ on $\partial Y$ pinched to rank-1 cusps. The fact that such a structure exists follows as a consequence of Thurston's uniformization theorem (see Morgan [22]).

As in the proof of Theorem 4.7, glue $H_{0}$ to $C_{0}$ by isometry, and then Dehn fill $P$ along slopes of the form $\mu_{i}+k \lambda_{i}$. Any such Dehn filling along $P$ fixes the disk set of $Y$, and modifies the disk set of $C_{0}$ by applying multitwist along $P$. By choice of $Y$ and Lemma 4.11, any such Dehn filling will yield a manifold with large Hempel distance, larger than $N-1>2 n+2$. Then work of Scharlemann and Tomova implies that the minimal genus Heegaard splitting must have genus at least $(n+1)$, which means that the manifold is tunnel number $n$.

On the other hand, the same proof as that of Theorem 4.7 applies to show that $n-1$ tunnels are arbitrarily close to self-intersecting.

\section{References}

[1] C C Adams, Unknotting tunnels in hyperbolic 3-manifolds, Math. Ann. 302 (1995) 177-195 MR1329452

[2] C C Adams, A W Reid, Unknotting tunnels in two-bridge knot and link complements, Comment. Math. Helv. 71 (1996) 617-627 MR1420513

[3] H Akiyoshi, M Sakuma, M Wada, Y Yamashita, Jørgensen's picture of punctured torus groups and its refinement, from: "Kleinian groups and hyperbolic 3-manifolds", (Y Komori, V Markovic, C Series, editors), London Math. Soc. Lecture Note Ser. 299, Cambridge Univ. Press (2003) 247-273 MR2044553

[4] B H Bowditch, Geometrical finiteness for hyperbolic groups, J. Funct. Anal. 113 (1993) 245-317 MR1218098

[5] J F Brock, K W Bromberg, On the density of geometrically finite Kleinian groups, Acta Math. 192 (2004) 33-93 MR2079598

[6] S D Burton, Unknotting tunnels of hyperbolic tunnel number $n$ manifolds, master's thesis, Brigham Young University (2012)

[7] R D Canary, M Culler, S Hersonsky, P B Shalen, Approximation by maximal cusps in boundaries of deformation spaces of Kleinian groups, J. Differential Geom. 64 (2003) 57-109 MR2015044 
[8] D Cooper, D Futer, J S Purcell, Dehn filling and the geometry of unknotting tunnels, Geom. Topol. 17 (2013) 1815-1876 MR3073937

[9] D Cooper, M Lackenby, J S Purcell, The length of unknotting tunnels, Algebr. Geom. Topol. 10 (2010) 637-661 MR2606795

[10] M Culler, P B Shalen, Varieties of group representations and splittings of 3-manifolds, Ann. of Math. 117 (1983) 109-146 MR683804

[11] D B A Epstein, C Petronio, An exposition of Poincaré's polyhedron theorem, Enseign. Math. 40 (1994) 113-170 MR1279064

[12] J Hempel, 3-manifolds as viewed from the curve complex, Topology 40 (2001) 631657 MR1838999

[13] T Jørgensen, On cyclic groups of Möbius transformations, Math. Scand. 33 (1973) 250-260 MR0348103

[14] T Jørgensen, On pairs of once-punctured tori, from: "Kleinian groups and hyperbolic 3-manifolds", (Y Komori, V Markovic, C Series, editors), London Math. Soc. Lecture Note Ser. 299, Cambridge Univ. Press (2003) 183-207 MR2044551

[15] M Lackenby, J S Purcell, Geodesics and compression bodies, to appear in Experimental Mathematics arXiv:1302.3652

[16] W B R Lickorish, A representation of orientable combinatorial 3-manifolds, Ann. of Math. 76 (1962) 531-540 MR0151948

[17] J Maher, S Schleimer, The handlebody complex, in preparation

[18] A Marden, The geometry of finitely generated kleinian groups, Ann. of Math. 99 (1974) 383-462 MR0349992

[19] A Marden, Outer circles: An introduction to hyperbolic 3-manifolds, Cambridge Univ. Press (2007) MR2355387

[20] B Maskit, Kleinian groups, Grundl. Math. Wissen. 287, Springer, Berlin (1988) MR959135

[21] C McMullen, Cusps are dense, Ann. of Math. 133 (1991) 217-247 MR1087348

[22] J W Morgan, On Thurston's uniformization theorem for three-dimensional manifolds, from: "The Smith conjecture", (J W Morgan, H Bass, editors), Pure Appl. Math. 112, Academic Press, Orlando, FL (1984) 37-125 MR758464

[23] D Rolfsen, Knots and links, Mathematics Lecture Series 7, Publish or Perish, Houston, TX (1990) MR1277811

[24] M Scharlemann, Heegaard splittings of compact 3-manifolds, from: "Handbook of geometric topology", (R J Daverman, R B Sher, editors), North-Holland, Amsterdam (2002) 921-953 MR1886684

[25] M Scharlemann, M Tomova, Alternate Heegaard genus bounds distance, Geom. Topol. 10 (2006) 593-617 MR2224466 
[26] W P Thurston, The geometry and topology of 3-manifolds, lecture notes, Princeton University (1978-1981) Available at http://library.msri.org/books/gt3m

[27] M Wada, Opti, a program to visualize quasi-conformal deformations of once-punctured torus groups Available at http://vivaldi.ics.nara-wu.ac.jp/ wada/OPTi

Department of Mathematics, Michigan State University

East Lansing, MI 48824, USA

Department of Mathematics, Brigham Young University

Provo, UT 84602-6539, USA

burtons8@msu.edu, jpurcell@math.byu.edu

Received: 12 March 2013 Revised: 2 August 2013 